Pacific Journal of Mathematics

CONVERGENCE OF A SEQUENCE OF TRANSFORMATIONS 


\title{
CONVERGENCE OF A SEQUENCE OF TRANSFORMATIONS OF DISTRIBUTION FUNCTIONS
}

\author{
W. L. Harkness and R. Shantaram
}

Let $F$ be the distribution function (d.f.) of a nonnegative random variable (r.v.) $X$ all of whose moments $\mu_{n}=\int_{0}^{\infty} x^{n} d F(x)$ exist and are finite. Define, recursively, the sequence $\left\{G_{n}\right\}$ of absolutely continuous d.f.'s as follows: put

$$
\begin{aligned}
& G_{1}(x)=\mu_{1}^{-1} \int_{0}^{x}[1-F(y)] d y \text { for } x>0 \text { and } \\
& G_{1}(x)=0 \text { for } x \leq 0 ; \text { for } n>1, \text { let } \\
& G_{n}(x)=\mu_{1, n-1}^{-1} \int_{0}^{x}\left[1-G_{n-1}(y)\right] d y \text { for } x>0 \text { and } \\
& G_{n}(x)=0 \text { for } x \leq 0, \text { where } \\
& \mu_{1, n-1}=\int_{0}^{\infty}\left[1-G_{n-1}(y)\right] d y .
\end{aligned}
$$

It is shown that if $F$ is distributed on a finite interval, then the sequence $\left\{G_{n}(x / n)\right\}$ converges to the simple exponential d.f. On the other hand, if $F(x)<1$ for all $x>0$ and $G_{n}\left(c_{n} x\right) \rightarrow G(x)$, where $G$ is a proper d.f. and $\left\{c_{n}\right\}$ is a sequence of constants such that $\left\{c_{n} / c_{n-1}\right\}$ is bounded, then (among other things) it is shown that (a) the convergence is uniform, (b) $G$ is continuous and concave on $[0, \infty),(\mathbf{c}) c_{n}$ is asymptotically equal to $\mu_{n+1} / b(n+1) \mu_{n}$ where $b=\int_{0}^{\infty}[1-G(u)] d u$ and (d) $\lim c_{n} / c_{n-1}$ exists. Finally, criteria for the existence of a sequence $\left\{c_{n}\right\}$ such that $\left\{G_{n}\left(c_{n} x\right)\right\}$ converges to a proper d.f. are given. In particular, it is shown that this sequence converges if $F$ is absolutely continuous with probability density function (p.d.f.) $f$ and $F$ has increasing hazard rate.

The d.f. $G_{1}$ is obtained as an (integral) transform of the d.f. $F$. These transforms (although not explicitly labeled as such) have been encountered frequently in renewal theory. In particular, it is such a transform (d.f.) which, in a delayed renewal process, makes the renewal rate a constant. In order to verify that $G_{1}$ (and hence, inductively, $G_{n}$ for $n>1$ ) is indeed a d.f. one makes use of the well-known alternative computational formula for the mean $\mu_{1}$ of a nonnegative r.v. $X$ with d.f. $F$ :

$$
\mu_{1}=\int_{0}^{\infty} x d F(x)=\int_{0}^{\infty}[1-F(x)] d x
$$

More generally, it may be shown that (cf. Feller [3], p. 148) 


$$
\mu_{n}=\int_{0}^{\infty} x^{n} d F(x)=n \int_{0}^{\infty} x^{n-1}[1-F(x)] d x .
$$

A d.f. $F$ is said to be a finite d.f. if there exists real numbers $a$ and $b$ such that $F(x)=0$ for $x \leqq a$ and $F(x)=1$ for $x \geqq b$. The d.f. $G$, given by $G(x)=1-e^{-b x}$ for $x>0$ and zero for $x \leqq 0$, with $b>0$, is called the exponential d.f. The c.f. $\varphi$ of a d.f. $F$, defined for all real $t$ by $\varphi(t)=\int_{-\infty}^{\infty} e^{i t x} d F(x)$, is said to be an analytic c.f. if $\varphi(t)$ coincides in some (real) neighborhood of $t=0$ with an analytic function $A(z)$ of a complex variable $z=t+i v$. In particular, if $A(z)$ is entire then $\varphi$ is called an entire c.f.

In $\S 2$ we calculate explicitly the moments and c.f. of $G_{n}, n \geqq 1$. We prove in $\S 3$ that for a finite d.f. $F$ the sequence $\left\{G_{n}(x / n)\right\}$ converges to the exponential d.f. The following three sections are devoted to the problem of existence of a sequence $\left\{c_{n}\right\}$ of constants such that the sequence $\left\{G_{n}\left(c_{n} x\right)\right\}$ converges to a proper d.f. Finally, in the last section some examples are given.

2. Moments, characteristic functions. Throughout $F$ will be a d.f. of a nonnegative r.v., all of whose moments $\mu_{n}$ are finite. Let $\varphi_{F}$ and $\varphi_{G_{n}}$ denote the c.f.'s of $F$ and $G_{n}$, respectively; the $k^{\text {th }}$ moment of $G_{n}$ will be denoted by $\mu_{k, n}$. We first consider the case $n=1$.

THEOREM 2.1. The c.f. $\varphi_{G_{1}}$ of $G_{1}$ is given by

$$
\varphi_{G_{1}}(t)=\left(i t \mu_{1}\right)^{-1}\left[\varphi_{F}(t)-1\right], \text { for } t \neq 0 \text {, and } \varphi_{G_{1}}(0)=1 \text {. }
$$

Proof. Clearly, $\varphi_{G_{1}}(0)=1$. For $t \neq 0$,

$$
\begin{aligned}
\varphi_{G_{1}}(t) & =\int_{0}^{\infty} e^{i t x} d G_{1}(x)=\mu_{1}^{-1} \int_{0}^{\infty} e^{i t x}[1-F(x)] d x \\
& =\mu_{1}^{-1} \lim _{a \rightarrow \infty} \int_{0}^{a} e^{i t x}[1-F(x)] d x \\
& =\lim _{a \rightarrow \infty}\left(i t \mu_{1}\right)^{-1}\left\{e^{i t a}[1-F(a)]+\int_{0}^{a} e^{i t x} d F(x)-1\right\} \\
& =\left(i t \mu_{1}\right)^{-1}\left[\varphi_{F}(t)-1\right] .
\end{aligned}
$$

THEOREM 2.2. The moments $\mu_{k, 1}$ of $G_{1}$ are given by

$$
\mu_{k, 1}=\mu_{k+1} /(k+1) \mu_{1}, \quad k=1,2, \cdots .
$$

Proof. By definition,

$$
\begin{aligned}
\mu_{k, 1} & =\int_{0}^{\infty} x^{k} d G_{1}(x)=\mu_{1}^{-1} \int_{0}^{\infty} x^{k}[1-F(x)] d x \\
& =\mu_{k+1} /(k+1) \mu_{1}
\end{aligned}
$$


using (1.1).

Using the recurrence relation

$$
\begin{aligned}
\mu_{k, n} & =\int_{0}^{\infty} x^{k} d G_{n}(x)=\mu_{1, n-1}^{-1} \int_{0}^{\infty} x^{k}\left[1-G_{n-1}(x)\right] d x \\
& =\mu_{k+1, n-1} /(k+1) \mu_{1, n-1}
\end{aligned}
$$

and mathematical induction, we obtain the following result.

Theorem 2.3. For all positive integers $n$ and $k$,

$$
\mu_{k, n}=k ! n ! \mu_{n+k} /(n+k) ! \mu_{n}=\left(\begin{array}{c}
n+k \\
k
\end{array}\right)^{-1} \mu_{n}^{-1} \mu_{n+k} .
$$

The result given in Theorem 2.3 is of some independent interest, inasmuch as it asserts that if $\left\{\mu_{n}\right\}$ is the moment sequence of a nonnegative r.v. then the sequence $\left\{\mu_{k, n}\right\}$, given in (2.2), is also a moment sequence for every $n$.

Similarly, using the recurrence relation (for $t \neq 0$ )

$$
\varphi_{G_{n}}(t)=\left(i t \mu_{1, n-1}\right)^{-1}\left[\varphi_{G_{n-1}}(t)-1\right],
$$

obtained by replacing $G_{1}$ by $G_{n}$ and $F$ by $G_{n-1}$ in Theorem 2.1, and induction, we can prove the following theorem, which again is mostly of independent interest.

THeOREM 2.4. The c.f. of $G_{n}$ is given by

$$
\varphi_{G_{n}}(t)=\frac{n !}{\mu_{n}(i t)^{n}}\left[\varphi_{F}(t)-\sum_{j=0}^{n-1} \mu_{j} \frac{(i t)^{j}}{j !}\right], t \neq 0
$$

with $\varphi_{G_{n}}(0)=1$.

Finally, we observe that if $\left\{c_{n}\right\}$ is a sequence of positive real numbers then $\left\{H_{n}\right\}, H_{n}=G_{n}\left(c_{n} x\right)$, is a sequence of d.f.'s, with the $k^{\text {th }}$ moment of $H_{n}$ being given by

$$
\mu_{k}\left(H_{n}\right)=\mu_{k, n} / c_{n}^{k} .
$$

3. Convergence of $\left\{G_{n}\right\}$ for finite d.f.'s. We shall prove in this section that for finite d.f.'s $\lim _{n \rightarrow \infty} G_{n}(x / n)$ is an exponential d.f. We need some lemmas.

Lemma 3.1. If all the moments $\mu_{n}$ of $F$ are finite then $\left\{\mu_{n+1} / \mu_{n}\right\}$ is a monotonically nondecreasing sequence.

Proof. For $n$ a positive integer and $t$ real, 


$$
0 \leqq \int_{0}^{\infty}\left(x^{(n+1) / 2}+t x^{(n-1) / 2}\right)^{2} d F(x)=\mu_{n+1}+2 t \mu_{n}+t^{2} \mu_{n-1} .
$$

Hence $\mu_{n}^{2}-\mu_{n+1} \mu_{n-1} \leqq 0$ implying that $\mu_{n} \mu_{n-1} \leqq \mu_{n+1} / \mu_{n}, n \geqq 1$.

CoRoLlaRY. $\quad \mu_{n+k} / \mu_{n} \geqq\left(\mu_{n+1} / \mu_{n}\right)^{k}, n, k \geqq 1$.

The following result is easily proved (see Boas [2]).

LEMmA 3.2. If $F$ is a finite d.f. on $[a, b]$ where $0 \leqq a<b<\infty$ and $b=\inf \{x \mid F(x)=1\}$ then $\lim _{n \rightarrow \infty} \mu_{n}^{1 / n}=b$.

LEMMA 3.3. Under the hypothesis of Lemma 3.2

$$
\lim _{n \rightarrow \infty} \mu_{n+k} / \mu_{n}=b^{k}, k \geqq 0 .
$$

Proof. Since $\left\{\mu_{n+1} / \mu_{n}\right\}$ is a nondecreasing sequence $\lim _{n-\infty} \mu_{n+1} / \mu_{n}=$ $L \leqq \infty$ exists. By a well-known theorem this implies that $\lim _{n \rightarrow \infty} \mu_{n}^{1 / n}=$ $L$ and by Lemma 3.2 it follows that $L=b<\infty$. Hence

$$
\lim _{n \rightarrow \infty} \mu_{n+k} / \mu_{i n}=\left(\lim _{n \rightarrow \infty} \mu_{n+1} / \mu_{n}\right)^{k}=L^{k}=b^{k} .
$$

The lemma is proved.

Theorem 3.1. Let $F$ be a finite d.f. on $[a, b]$, where

$$
b=\inf \{x \mid F(x)=1\} .
$$

Then $\lim _{n \rightarrow \infty} G_{n}(x / n)=G(x) \equiv 1-e^{-x / b}$ for $x>0$ and zero for $x \leqq 0$.

Proof. Letting $c_{n}=1 / n$ we get from (2.2) and (2.4) that $\mu_{k}\left(H_{n}\right)=$ $n^{k} \mu_{k, n} \rightarrow k ! b^{k}$ as $n \rightarrow \infty$. The limit is the $k^{\text {th }}$ moment of the exponential d.f. $G(x)$ given in the statement of the theorem. The present theorem new follows from the moment convergence theorem (cf. Loeve [7], p. 185).

4. D.F.'s on an infinite range. For finite d.f.'s Theorem 3.1 gives an explicit sequence $\left\{c_{n}\right\}$, namely, $c_{n}=1 / n$, of normalizers such that $G_{n}\left(c_{n} x\right)$ converges to an exponential d.f. We investigate, in the remainder of this paper the problem of existence of the sequence $\left\{c_{n}\right\}$ for d.f.'s $F$ on $[0, \infty)$ (i.e., $F(x)<1$ for all $x$ ) all of whose moments $\mu_{n}$ are finite. Henceforth $F$ will stand for such a d.f.

Theorem 4.1. $\lim _{n \rightarrow \infty} \mu_{n+1} / \mu_{n}=+\infty$

Proof. Since $\left\{\mu_{n+1} / \mu_{n}\right\}$ is nondecreasing its limit $L \leqq+\infty$ exists. Assume to the contrary that $L<+\infty$. This implies $\lim _{n \rightarrow \infty} \mu_{n}^{1 / n}=$ $L<\infty$. We now show that this is a contradiction by proving that $\lim _{n \rightarrow \infty} \mu_{n}^{1 / n}=+\infty$. Let $A>0$ be an arbitrary (fixed) number. Then 


$$
\mu_{n}=\int_{0}^{\infty} x^{n} d F(x) \geqq \int_{A}^{\infty} x^{n} d F(x) \geqq A^{n}[1-F(A)] .
$$

Hence, $\lim _{n \rightarrow \infty}$ inf $\mu_{n}^{1 / n} \geqq A$. Since $A$ was arbitrary we conclude that $\mu_{n}^{1 / n} \rightarrow+\infty$ as $n \rightarrow \infty$. Hence $L=+\infty$.

We shall need the following result which is easily proven by induction on $k$.

LEMMA 4.2. If $\lim \mu_{n+2} \mu_{n} / \mu_{n+1}^{2}=l<\infty$ then for each $k \geqq 0$ and $0 \leqq r \leqq k, \lim _{n \rightarrow \infty} \mu_{n+k} \mu_{n} / \mu_{n+r} \mu_{n+k-r}=l^{r(k-r)}$.

LeMmA 4.3. If $F(x)$ has an analytic c.f., $\lim \inf _{n \rightarrow \infty} \mu_{n+2} \mu_{n} / \mu_{n+1}^{2}=1$.

Proof. By Lemma 3.1, $\lim \inf _{n \rightarrow \infty} \mu_{n+2} \mu_{n} / \mu_{n+1}^{2}=\alpha \geqq 1$. If $\alpha>1$, choose $\beta$ such that $\alpha>\beta>1$. Then there exists $N$ such that

$$
\mu_{n+2} \mu_{n} / \mu_{n+1}^{2}>\beta
$$

for $n \geqq N$. Hence $\mu_{N+2} \mu_{N} / \mu_{N+1}^{2}>\beta$; i.e., $\mu_{N+2}>\beta \mathbf{a}_{N} b_{N}$ where $a_{N}=$ $\mu_{N+1} / \mu_{N}$ and $b_{N}=\mu_{N+1}$. Similarly, $\mu_{N+3}>\beta^{3} a_{N}^{2} b_{N}$, and, by induction, it follows that $\mu_{N+k+1}>\beta^{k(k+1) / 2} a_{N}^{k} b_{N}, k \geqq 1$. Letting $N+k+1=n$ this becomes $\mu_{n}>\beta^{(n-N)(n-N-1) / 2} a_{N}^{n-N-1} b_{N}$ for $n \geqq N+2$ and so

$$
\limsup _{n \rightarrow \infty} \mu_{n}^{1 / n} / n \geqq \limsup _{n \rightarrow \infty} \beta^{(n-N-1) / 2} a_{N / n}=+\infty
$$

since $\beta>1$. This is a contradiction since lim $\sup _{n \rightarrow \infty} \mu_{n}^{1 / n} / n<\infty$ if $F(x)$ has an analytic c.f. (see [8] p. 136). The lemma is proved.

The main theorem of this section is the following.

THEOREM 4.1. Let $\left\{c_{n}\right\}$ be a sequence of positive real numbers such that $H_{n}(x) \equiv G_{n}\left(c_{n} x\right) \rightarrow G(x)$, with $G(x)$ a proper d.f. and assume that $\lim \sup _{n \rightarrow \infty} c_{n} / c_{n-1}=l<\infty$. Then

( i ) $\left\{b_{n}\right\}$ is a bounded sequence where $b_{n}=\int_{0}^{\infty}\left[1-H_{n}(u)\right] d u$.

(ii ) $b=\int_{0}^{\infty}[1-G(u)] d u<\infty$.

(iii) $b_{n} \rightarrow b$ as $n \rightarrow \infty$.

(iv) $\lim _{n \rightarrow \infty} c_{n} / c_{n-1}=l$.

( v ) If $\lim _{n \rightarrow \infty} a_{n}=\lambda$ where $a_{n}>0, \lambda>0$ then $\lim _{n \rightarrow \infty} H_{n}\left(a_{n} x\right)=$ $G(\lambda x)$.

(vi ) $G(x)$ is continuous and concave on $[0, \infty)$ and $\lim _{n \rightarrow \infty} H_{n}^{\prime}(x)=$ $G^{\prime}(x)$ for $x>0$.

(vii) $l \geqq 1$ and equality holds if $F$ has an analytic c.f.

(viii) $\lim _{n \rightarrow \infty} \mu_{k}\left(H_{n}\right)=\mu_{k}(G)$ for $k \geqq 0$, where $\mu_{k}(G)$ is the $k^{\text {th }}$ moment of $G$. 
Proof. Let $b_{n}=\int_{0}^{\infty}\left[1-H_{n}(u)\right] d u$ and $b=\int_{0}^{\infty}[1-G(u)] d u$.

(i) Since $\lim \sup _{n \rightarrow \infty} c_{n} / c_{n-1}=l<\infty$, there exists $N^{\prime}$ such that $c_{n} / c_{n-1}<l+1$ for $n>N^{\prime}$. Also there exists $x_{0}$ and $N^{\prime \prime}$ such that $G\left(x_{0}\right)>\frac{1}{2}$ and $H_{n}\left(x_{0}\right)>\frac{1}{4}$ for $n>N^{\prime \prime}$. This last assertion is valid since $H_{n}\left(x_{0}\right) \rightarrow G\left(x_{0}\right)>\frac{1}{2}$. Now

$$
\begin{aligned}
H_{n}(x) & =G_{n}\left(c_{n} x\right)=\int_{0}^{c_{n} x}\left[1-G_{n-1}(u)\right] d u / \int_{0}^{\infty}\left[1-G_{n-1}(u)\right] d u \\
& =b_{n-1}^{-1} \int_{0}^{c_{n} x / c_{n-1}}\left[1-H_{n-1}(u)\right] d u
\end{aligned}
$$

by a simple change of variable. For $n>\max \left(N^{\prime}, N^{\prime \prime}\right)$ (4.1) yields

$$
H_{n}\left(x_{0}\right) \leqq b_{n-1}^{-1} \int_{0}^{(l+1) x_{0}}\left[1-H_{n-1}(u)\right] d u
$$

Hence

$$
\begin{aligned}
\limsup _{n \rightarrow \infty} b_{n-1} \leqq \limsup _{n \rightarrow \infty} \int_{0}^{(l+1) x_{0}}\left[1-H_{n-1}(u)\right] d u / H_{n}\left(x_{0}\right) \\
=\int_{0}^{(l+1) x_{0}}[1-G(u)] d u / G\left(x_{0}\right)<\infty .
\end{aligned}
$$

(ii) By Fatou's lemma and (4.2)

$$
b=\int_{0}^{\infty}[1-G(u)] d u \leqq \liminf _{n \rightarrow \infty} \int_{0}^{\infty}\left[1-H_{n}(u)\right] d u<\infty .
$$

(iii) Let $\sup _{n} b_{n}=D<\infty$ and $\sup _{n} c_{n} / c_{n-1}=M<\infty$. From (4.1),

$$
1-H_{n}(x)=b_{n-1}^{-1} \int_{c_{n} x / c_{n-1}}^{\infty}\left[1-H_{n-1}(u)\right] d u,
$$

so that for $n \geqq 2$ and $x \geqq 0$

$$
\begin{aligned}
& \int_{M x}^{\infty}\left[1-H_{n-1}(u)\right] d u \leqq \int_{c_{n} x / c_{n-1}}^{\infty}\left[1-H_{n-1}(u)\right] d u \\
& \quad=b_{n-1}\left[1-H_{n}(x)\right] \leqq D\left[1-H_{n}(x)\right] .
\end{aligned}
$$

Now let $\varepsilon>0$ be arbitrary. Pick $x_{0}$ such that $1-G\left(x_{0}\right)<\varepsilon / 2 D$ and pick $x_{1}>M x_{0}$ such that $\int_{x_{1}}^{\infty}[1-G(u)] d u<\varepsilon$. Then there exists $N$ such that for $n>N, 1-H_{n}\left(x_{0}\right)<\varepsilon / D$ and also

$$
\left|\int_{0}^{x_{1}}\left[1-H_{n-1}(u)\right] d u-\int_{0}^{x_{1}}[1-G(u)] d u\right|<\varepsilon \text {. }
$$

Since (4.3) holds for all $x$,

$$
\int_{x_{1}}^{\infty}\left[1-H_{n-1}(u)\right] d u \leqq \int_{M x_{0}}^{\infty}\left[1-H_{n-1}(u)\right] d u \leqq D\left[1-H_{n}\left(x_{0}\right)\right]<\varepsilon .
$$

Then for $n>N$, 


$$
\begin{aligned}
& \left|\int_{0}^{\infty}\left[1-H_{n-1}(u)\right] d u-\int_{0}^{\infty}[1-G(u)] d u\right| \\
& \leqq \\
& \quad\left|\int_{0}^{x_{1}}\left[1-H_{n-1}(u)\right] d u-\int_{0}^{x_{1}}[1-G(u)] d u\right| \\
& \quad+\int_{x_{1}}^{\infty}\left[1-H_{n-1}(u)\right] d u+\int_{x_{1}}^{\infty}[1-G(u)] d u<3 \varepsilon .
\end{aligned}
$$

Hence $b_{n} \rightarrow b$ as $n \rightarrow \infty$. In other words the sequence of first moments of $G_{n}\left(c_{n} x\right)$ converges to the first moment of $G(x)$. Recalling (2.2) and (2.4) we conclude that $c_{n} \sim \mu_{n+1} / b(n+1) \mu_{n}$. Note that $b>0$ since $G(x)$ is a proper d.f.

(iv) Since $\left\{c_{n} / c_{n-1}\right\}$ is bounded the sequence will fail to have a limit only if $\infty>\lim \sup _{n \rightarrow \infty} c_{n} / c_{n-1}=p>q=\liminf _{n \rightarrow \infty} c_{n} / c_{n-1}$. It suffices to assume $p>0$ and $p>q$ and obtain a contradiction.

Let $0<\varepsilon<(p-q) / 2$. Then $p-\varepsilon>q+\varepsilon$. Let $\left\{c_{n^{\prime}} / c_{n^{\prime}-1}\right\}$ be a subsequence converging to $p$ and let $c_{n^{\prime}} / c_{n^{\prime}-1}>p-\varepsilon$ for $n^{\prime}>N$. Then, from (4.1) for $x>0$ and $n^{\prime}>N$ we have

$$
H_{n^{\prime}}(x) \geqq b_{n^{\prime}-1}^{-1} \int_{0}^{(p-\varepsilon) x}\left[1-H_{n^{\prime}-1}(u)\right] d u
$$

so that $G(x) \geqq b^{-1} \int_{0}^{(p-s) x}[1-G(u)] d u$. In a like manner,

$$
G(x) \leqq b^{-1} \int_{0}^{(q+\varepsilon) x}[1-G(u)] d u .
$$

Since $p-\varepsilon>q+\varepsilon$ these inequalities together imply that

$$
\int_{(q+\odot) x}^{(p-s) x}[1-G(u)] d u=0
$$

or that $G(u)=1$ a.e. on $[(q+\varepsilon) x,(p-\varepsilon) x]$. If $G(x)$ is continuous on $(0, \infty)$ it would follow that $G(u)=1$ on $[(q+\varepsilon) x,(p-\varepsilon) x]$ for $x>0$ and so $G(x)$ is degenerate at the origin which is a contradiction of our hypothesis. Hence $p=q$ and $\lim _{n \rightarrow \infty} c_{n} / c_{n-1}=l$ exists. Hence it suffices to show that $G(x)$ is continuous on $(0, \infty)$. It is clear that $G_{n}(x)$ has derivatives for $x>0$ up to order $n-1$. Therefore, for $n \geqq 3$. we obtain from (4.1) that

$$
\begin{gathered}
H_{n}^{\prime}(x)=\left(c_{n} / c_{n-1}\right)\left[1-G_{n-1}\left(c_{n} x\right)\right] / b_{n-1} \geqq 0 \\
H_{n}^{\prime \prime}(x)=-c_{n}^{2}\left[1-G_{n-2}\left(c_{n} x\right)\right] / c_{n-1} c_{n-2} b_{n-1} b_{n-2} \leqq 0
\end{gathered}
$$

for $x>0$. Hence $H_{n}(x)$ is a concave d.f. for $n \geqq 3$ and $x>0$. So

$$
\begin{aligned}
& G(\theta x+(1-\theta) y)=\lim _{n \rightarrow \infty} H_{n}(\theta x+(1-\theta) y) \\
& \quad \geqq \lim _{n \rightarrow \infty}\left[\theta H_{n}(x)+(1-\theta) H_{n}(y)\right]=\theta G(x)+(1-\theta) G(y)
\end{aligned}
$$


and $G(x)$ is concave for $x>0$ and hence continuous on $(0, \infty)$.

( $\mathrm{v}$ ) Let $\varepsilon>0$ be given and let $x>0$. Let $a_{n}>0$ and $a_{n} \rightarrow \lambda>0$. Since $G$ is continuous at $\lambda x$, there is a $\delta>0$ such that

$$
|G(\lambda x+h)-G(\lambda x)|<\varepsilon \text { for }|h|<\delta .
$$

Pick $\varepsilon^{\prime}$ such that $0<\varepsilon^{\prime}<\min (\varepsilon, \delta / x)$. Then for $n$ large $H_{n}\left(a_{n} x\right)-$ $H_{n}(\lambda x)<H_{n}\left(\left(\lambda+\varepsilon^{\prime}\right) x\right)-H_{n}(\lambda x) \rightarrow G\left(\lambda x+\varepsilon^{\prime} x\right)-G(\lambda x)<\varepsilon$. Hence for large enough $n, H_{n}\left(a_{n} x\right)-H_{n}(\lambda x)<2 \varepsilon$. Similarly, for large enough $n$, $H_{n}\left(a_{n} x\right)-H_{n}(\lambda x)>-2 \varepsilon$. Thus

$$
\begin{gathered}
\left|H_{n}\left(a_{n} x\right)-G(\lambda x)\right| \leqq\left|H_{n}\left(a_{n} x\right)-H_{n}(\lambda x)\right| \\
\quad+\left|H_{n}(\lambda x)-G(\lambda x)\right|<2 \varepsilon+\varepsilon=3 \varepsilon
\end{gathered}
$$

for sufficiently large $n$. That is, $\lim _{n \rightarrow \infty} H_{n}\left(a_{n} x\right)=G(\lambda x)$.

( vi ) By virtue of (4.4) and (iii), (iv), and (v),

$$
H_{n}^{\prime}(x) \rightarrow l b^{-1}[1-G(l x)]
$$

for $x>0$. On the other hand we see from (4.1) that

$$
G(x)=b^{-1} \int_{0}^{l x}[1-G(u)] d u, x>0
$$

and so for $x>0$

$$
G^{\prime}(x)=l b^{-1}[1-G(l x)]=\lim _{n \rightarrow \infty} H_{n}^{\prime}(x) .
$$

Since $G(x)$ is continuous it follows from (4.5) that $G^{\prime}(x)$ exists for all $x>0$.

It remains to show that $G(x)$ is continuous at the origin. Clearly, by definition, $G(0)=0$. Also, from (4.5), $G(x) \leqq l b^{-1} x<\varepsilon$ for $x>0$ sufficiently small. Together with the argument in (iv), this completes the proof that $G(x)$ is continuous and concave on $[0, \infty)$.

(vii) From the remark at the end of (iii) we know that $c_{n} \sim$ $\mu_{n+1} / b(n+1) \mu_{n}$. Since $c_{n} / c_{n-1} \rightarrow l$ it follows that $\mu_{n+2} / \mu_{n} / \mu_{n+1}^{2} \rightarrow l$. Hence $l \geqq 1$ (Lemma 3.1) and $l=1$ if $F$ has an analytic c.f. (Lemma 4.3).

(viii) Since $\mu_{n+2} \mu_{n} / \mu_{n+1}^{2} \rightarrow l$ by (vii) we have from Lemma 4.2 that

$$
\left(\mu_{n} / \mu_{n+1}\right)^{k} \mu_{n+k} / \mu_{n}=\prod_{i=1}^{k} \mu_{n+i} \mu_{n} / \mu_{n+i-1} \mu_{n+1} \rightarrow \prod_{i=1}^{k} l^{i-1}=l^{k(k-1) / 2} .
$$

Further, from (iii) $\mu_{n+1} /(n+1) c_{n} \mu_{n} \rightarrow b$. Hence

$$
\begin{aligned}
\mu_{k}\left(H_{n}\right)= & \left(\begin{array}{c}
n+k \\
n
\end{array}\right)^{-1} \mu_{n+k} / \mu_{n} c_{n}^{k} \\
& \sim k !\left(\mu_{n+1} /(n+1) c_{n} \mu_{n}\right)^{k}\left(\mu_{n} / \mu_{n+1}\right)^{k} \mu_{n+k} \mu_{n}, n \rightarrow \infty \\
& \rightarrow k ! b^{k} l^{k(k-1) / 2}=\nu_{k}, \quad \text { say. }
\end{aligned}
$$


The proof of (viii) would be completed on showing that $\nu_{k}$ is the $k^{\text {th }}$ moment of the limiting d.f. $G(x)$ and this we show now. From (4.5) $d G(x)=l b^{-1}[1-G(l x)] d x$ and hence by $(1.1)$

$$
\begin{aligned}
\mu_{k}(G) & =\int_{0}^{\infty} x^{k} d G(x)=k \int_{0}^{\infty} x^{k-1}[1-G(x)] d x \\
& =k b \int_{0}^{\infty} x^{k-1} d G(x / l)=k b l^{k-1} \mu_{k-1}(G) .
\end{aligned}
$$

It follows by induction that, for $k>0, \mu_{k}(G)=k ! b^{k} l^{k(k-1) / 2}=\nu_{k}$.

5. Remarks on Theorem 4.1. The above theorem yields among other things a complete picture about the sequence $\left\{c_{n}\right\}$ (when it exists satisfying the conditions of the theorem). It shows (a) that asymptotically $c_{n} \sim \mu_{n+1} / b(n+1) \mu_{n}$ thus bringing out explicitly the connection between the normalizers and the growth rates of moments; (b) that the limiting d.f. $G(x)$ is continuous everywhere, concave and differentiable on $(0, \infty)$ and has finite moments of all orders which are the limits of the corresponding moments of $G_{n}\left(c_{n} x\right)$; (c) that the convergence $G_{n}\left(c_{n} x\right) \rightarrow G(x)$ is uniform (cf. Parzen [9], p. 438) and (d) that the limiting d.f. satisfies the integral equation (4.5). The solutions of this equation will therefore yield the class of limit d.f.'s. This is an interesting equation and will be discussed elsewhere.

Note that if $l>1$ we can conclude from the form of $\nu_{k}$ that the limit d.f. is not exponential.

We close this section with the following simple result which is an easy consequence of Theorem 4.1.

THEOREM 5.1. Under the hypothesis of Theorem 4.1, $G(x)$ is an expotential d.f. provided that any one of the following equivalent conditions holds (a) $\lim \sup _{n \rightarrow \infty} c_{n}<\infty$ (b) $\mu_{n+1} / \mu_{n}=0(n)$.

6. An existence criterion for $\left\{c_{n}\right\}$. It was shown in Theorem 4.1 that if the $c_{n}$ are normalizers and $\lim \sup _{n \rightarrow \infty} c_{n} / c_{n-1}<\infty$ then $\lim _{n \rightarrow \infty} \mu_{n+2} \mu_{n} / \mu_{n+1}^{2}$ exists and is finite. In Theorem 6.1 below we show that this latter requirement is a sufficient condition for the existence of $\left\{c_{n}\right\}$ and in Theorem 6.2 we show that this condition is satisfied for a very wide class of distributions.

THeOREM 6.1. Let $F$ be a d.f. of a nonnegative r.V. having finite moments $\mu_{1}, \mu_{2}, \cdots$, such that $\delta_{n}=\mu_{n+2} \mu_{n} / \mu_{n+1}^{2} \rightarrow 1$ as $n \rightarrow \infty$. If $c_{n}=$ $\mu_{n+1} /(n+1) \mu_{n}$, then $G_{n}\left(c_{n} X\right) \rightarrow G(X)$, where $G(X)=1-e^{-X}$ for $X \geqq 0$ and $G(X)=0$ for $X \leqq 0$.

Proof. From the assumption $\delta_{n} \rightarrow 1$ as $n \rightarrow \infty$ it is clear that 
$c_{n} / c_{n-1} \rightarrow 1$. Furthermore, from the proof of Theorem 4.1 (iii), it follows that $\mu_{k}\left(H_{n}\right) \rightarrow k !$ as $n \rightarrow \infty$ for $k=1,2, \cdots$. The sequence $\{k !\}$ is a moment sequence uniquely determining the asserted d.f. $G$ and therefore the assertion of the theorem is a consequence of the moment convergence theorem.

In order to exhibit distributions for which $\mu_{n} \mu_{n+2} / \mu_{n+1}^{2}$ converges (to one) we need the following definitions and facts.

A function $g(x, y)$ of two real variables ranging over linearly ordered one-dimensional sets $\underline{\bar{X}}$ and $\underline{\bar{Y}}$, respectively, is said to be totally positive of order $r$ (denoted by $T P_{r}$ ) if for all $x_{1}<x_{2}<\cdots<x_{m}$, $y_{1}<y_{2}<\cdots<y_{m}$, with $x_{i} \in \underline{\bar{X}}, y_{j} \in \underline{\bar{Y}}$, and $m=1,2, \cdots, r$, the determinant of the $m \times m$ matrix with $(i, j)^{\text {th }}$ element $g\left(x_{i}, y_{j}\right)$ is nonnegative. If $g(x, y)$ is $T P_{r}$ for all $r$, this fact is indicated by saying $g(x, y)$ is $T P$. Also, a nonnegative function $k(x)$, defined for all real $x$, is said to be a Polya frequency function of order $r\left(P F_{r}\right)$ if $g(x, y)=k(x-y)$ is $T P_{r}$. In statistical applications, $y$ is usually a parameter and $g(x, y)$ is a probability density function (p.d.f.) in $x$ for each fixed $y$.

Following [1] we shall say that a d.f. $F$ has increasing hazard rate (IHR) if $\ln [1-F(x)]$ is concave (in which case the support $I$ of $F$ is an interval). The following results are well-known (see [1], [4], [5], and [6]). (6.1)

(i) $F$ has IHR if and onlf if $1-F$ is $P F_{2}$.

(ii) If $F$ is absolutely continuous with p.d.f. $f$, then $F$ has IHR if and only if the hazard function $q(x)=f(x) /[1-F(x)]$ is nondecreasing in $x \in I$.

(iii) The class $\mathscr{C}$ of all d.f.'s which have IHR is closed under convolution: $\quad F, G \in \mathscr{H} \Rightarrow H=F * G \in \mathscr{L}$.

(iv) Each member of the exponential family, a class of d.f.'s having p.d.f.'s of the form $k(x, y)=\beta(y) e^{x y}$ with respect to a $\sigma$-finite measure $\mu$ on $(-\infty, \infty)$, is TP. Here, $\underline{\bar{X}}=(-\infty, \infty)$ and

$$
\underline{\bar{Y}}=\left\{y: \beta(y)=\int e^{x y} d \mu(x)<\infty\right\}
$$

is an interval. The family includes the binomial, Poisson, gamma, and normal d.f.'s, for example.

(v) If $F$ has IHR, then $1-F(x)$ tends to zero as $x \rightarrow \infty$ exponentially fast. Thus, if $F$ has IHR or $1-F$ is $P F_{2}$, all moments of $F$ exist and are finite; in fact, $F$ has an analytic c.f.

(vi) $F$ and/or $1-F$ may be $P F_{2}$ while the p.d.f. $f$ (if it exists) is not $P F_{2}$. This is so even if $F$ has IHR. However, if $F$ is an absolutely continuous d.f. such that the p.d.f. $f$ is also $P F_{2}$, then $F$ has IHR.

(vii) If $G(y)=1-e^{-y}$ for $\mathrm{y} \geqq 0$ and zero elsewhere, and $F$ is a d.f. of a nonnegative r.v., then $F$ has IHR if and only if there exists 
a nonnegative convex increasing function $h$ such that $F(x)=G[h(x)]$. In [1], p. 384, it is shown that if $F(1)$ is absolutely continuous, (2) is the d.f. of a nonnegative r.v., and (3) has IHR, then for all real numbers $t>s>0$,

$$
\left(\lambda_{i+t} / \lambda_{i}\right)^{s} \leqq\left(\lambda_{i+s} / \lambda_{i}\right)^{t}
$$

where $\lambda_{r}=\mu_{r} / \Gamma(r+1)$ with $\mu_{r}=\int_{0}^{\infty} x^{r} d F(x)$. The inequality (6.2) was proven in [6] (cf., equation (16), p. 1032) for the special case $i=0$, assuming that $F$ has a continuous p.d.f. $f(x)$ which is $P F_{2}$. From (6.2), it follows immediately, on putting $i=n-1, t=2$, and $s=1$, that

$$
\left[\mu_{n+2} /(n+1)(n+2) \mu_{n}\right] \leqq\left[\mu_{n+1} /(n+1) \mu_{n}\right]^{2},
$$

i.e.,

$$
\mu_{n+2} / \mu_{n} / \mu_{n+1}^{2} \leqq 1+(n+1)^{-1} .
$$

Since $\mu_{n+2} / \mu_{n} / \mu_{n+1}^{2} \geqq 1$ always, we obtain the following theorem.

THEOREM 6.2. Let $F$ be the d.f. of a nonnegative r.v. and assume that $F$ has IHR and is absolutely continuous. Then

$$
\delta_{n}=\mu_{n} \mu_{n+2} / \mu_{n+1}^{2} \rightarrow 1, \quad \text { as } \quad n \rightarrow \infty .
$$

We remark that if $F$ is a finite d.f. on $[a, b], 0 \leqq a<b<\infty$, then by Lemma 3.3, $\delta_{n} \rightarrow 1$ as $n \rightarrow \infty$; no other assumptions are needed. Furthermore, all d.f.'s satisfying the hypotheses of Theorem 6.2 have analytic c.f.'s; an example in the following section shows that this is not necessary, so that Theorem 6.2 provides only sufficient conditions for $\delta_{n}$ to converge to unity.

7. Examples. This section contains several examples illustrating the results obtained in this paper. The first example illustrates Theorem 3.1; the second shows that analyticity is not necessary for the existence of normalizing constants $c_{n}$ such that $G_{n}\left(c_{n} x\right) \rightarrow G(x)$ with $\delta_{n} \rightarrow 1$; and the last few examples illustrate the concepts of $\S 6$ and Theorem 6.2.

EXAMPLE 1. Let $F(x)=1-p$ for $0<x \leqq 1,1$ for $x>1$ and zero elsewhere be the d.f. of the Bernoulli distribution $P(X=0)=1-p$, $P(X=1)=p, 0<p<1$, with mean $\mu_{1}=p$. Then $G_{n}(x)=1-(1-x)^{n}$ for $0 \leqq x \leqq 1,1$ for $x>1$ and zero elsewhere. $G_{n}(x)$ converges to the d.f. degenerate at the origin, but $\lim _{n-\infty} G_{n}(x / n)=G(x)=1-e^{-x}$ for $x>0$ and zero elsewhere. 
Example 2. $\mu_{n}=(4 n+3) ! / 6$ is the $n^{\text {th }}$ moment of a d.f. $F$ which is not uniquely determined by $\left\{\mu_{n}\right\}$ and hence does not have an analytic c.f. (cf., Widder [11], p. 126). But since

$$
\mu_{n+2} \mu_{n} / \mu_{n+1}^{2} \rightarrow 1, c_{n}=\mu_{n+1} /(n+1) \mu_{n} \sim 256 n^{3}
$$

are normalizing constants, i.e., $G_{n}\left(c_{n} x\right) \rightarrow G(x)$. Since $l=1$ the limit d.f. $G$ is exponential. (This can be varified by the moment convergence theorem also).

EXAMPLE 3. Let $F_{\beta}(x)=1-e^{-x^{\beta}}$, for $x>0, \beta>0$, and zero otherwise, be the d.f. of the Weibull distribution. The corresponding p.d.f. is given by $f_{\beta}(x)=\beta x^{\beta-1}\left[1-F_{\beta}(x)\right]$, so that the hazard function $q(x)=$ $\beta x^{\beta-1}$ is nondecreasing for $\beta \geqq 1$, so that (using 6.1 (ii)) $F$ has IHR for these values of $\beta$. By Theorem $6.2, \delta_{n} \rightarrow 1$ as $n \rightarrow \infty$, for $\beta \geqq 1$. By direct calculations it is easily seen that $\mu_{n}=\Gamma(n+\beta / \beta)$ and that $\delta_{n} \rightarrow 1$ for all $\beta>0$; clearly, the assumptions of Theorem 6.2 are not necessary.

EXAMPLE 4. Let $G_{\alpha, \beta}=F_{\alpha} * F_{\beta}$ be the convolution of two Weibull d.f.'s with parameters $\alpha$ and $\beta$. Then for $\alpha>1, \beta>1$, we conclude, using 6.1 (iii), that $G_{\alpha, \beta}$ has IHR, so that Theorem 6.2 applies. Verification of the fact that $\delta_{n} \rightarrow 1$ by computing the moments of $G_{\alpha, \beta}$ directly would appear to pose some difficulty.

EXAMPLE 5. For $x>0$, let $F_{1}(x)=1-e^{-x}=1-e^{-h_{0}(x)}, F_{2}(x)=$ $1-e^{-e^{x}}=1-e^{-h_{1}(x)}, \cdots, F_{k+1}(x)=1-e^{-h_{k}(x)}$, where $h_{0}(x)=x, h_{1}(x)=$ $e^{x}, \cdots, h_{k+1}(x)=e^{h_{k}(x)}$. It is readily seen that $h_{1}, \cdots, h_{k}$ are convex increasing functions, so that by 6.1 (vii) $F_{1}, \cdots, F_{k+1}$ have IHR's, and Theorem 6.2 again is applicable.

EXAMPLE 6. The p.d.f. $f(x, y)=e^{(x-y)-e^{(x-y)}}$, for

$$
x>y>0,0<y<\infty,
$$

belongs to the exponential family of distributions. Since $f(x, y)=$ $k(x-y)$, it is seen that $f$ is $T P$, i.e., $f$ is $P F$; Theorem 6.2 applies.

EXAMPLE 7. It is shown in [4] that

$$
f(x)=A \sum_{\nu=-\infty}^{\infty}(-1)^{\nu} e^{-\nu^{2} x}, x>0 ; 0 \text { for } x \leqq 0 .
$$

is $T P$, where $A$ is a normalizing constant. For suitable choice of $A$, $f$ is $P F_{r}$, for all $r$, i.e., $f$ is $P F$. More generally, each member of the family of p.d.f.'s given by $f(x-y), 0<y<\infty, x>y$, is $P F$. Therefore, the conclusion of Theorem 6.2 holds. 


\section{REFERENCES}

1. R. E. Barlow, A. W. Marshall, and F. Proschan, Properties of probability distributions with monotone hazard rate, Ann. Math. Stat. 34 (1963), 375-389.

2. R. P. Boas, Entire functions, Academic Press, New York, 1954.

3. W. Feller, An introduction to probability theory and its applications, Vol. 2, John Wiley and Sons, Inc., New York, 1966.

4. S. Karlin, Decision theory for Polya distributions, case of two actions, I, Proc. Third Berkeley Symp. on Probability and Statistics, Vol. 1, Univ. of California, Berkeley, 1956.

5. _ Total positivity, Stanford University Press, Stanford, 1968.

6. S. Karlin, F. Proschan, and R. E. Barlow, Moment inequalities of Polya frequency functions, Pacific J. Math. 11 (1961), 1023-33.

7. M. Loeve, Probability theory, D. Van Nostrand Company, Inc., Princeton, New Jersey, 1963.

8. E. Lukacs, Characteristic functions, Hafner Publishing Company, New York, 1960.

9. E. Parzen, Modern probability theory and its applications, John Wiley and Sons, Inc., New York, 1960.

10. G. Polya, Remarks on characteristic functions, Proc. Berkeley symp. on Math. Statist. and Prob., Univ. of California, Berkeley, 1949.

11. D. V. Widder, The laplace transform, Princeton University Press, Princeton, New Jersey, 1941.

Received May 11, 1967, and in revised form May 1, 1969.

The State University of New York at Stony Brook and

The Pennsylvania State University 



\section{PACIFIC JOURNAL OF MATHEMATICS}

\section{EDITORS}

\author{
H. ROYDEN \\ Stanford University \\ Stanford, California \\ Richard Pierce \\ University of Washington \\ Seattle, Washington 98105
}

\author{
J. DugundJI \\ Department of Mathematics \\ University of Southern California \\ Los Angeles, California 90007 \\ BASIL GoRDON \\ University of California \\ Los Angeles, California 90024
}

\section{ASSOCIATE EDITORS}
E. F. BECKENBACH
B. H. Neumann
F. WOLF
K. YoSHIDA

\section{SUPPORTING INSTITUTIONS}

\author{
UNIVERSITY OF BRITISH COLUMBIA \\ CALIFORNIA INSTITUTE OF TECHNOLOGY \\ UNIVERSITY OF CALIFORNIA \\ MONTANA STATE UNIVERSITY \\ UNIVERSITY OF NEVADA \\ NEW MEXICO STATE UNIVERSITY \\ OREGON STATE UNIVERSITY \\ UNIVERSITY OF OREGON \\ OSAKA UNIVERSITY \\ UNIVERSITY OF SOUTHERN CALIFORNIA
}

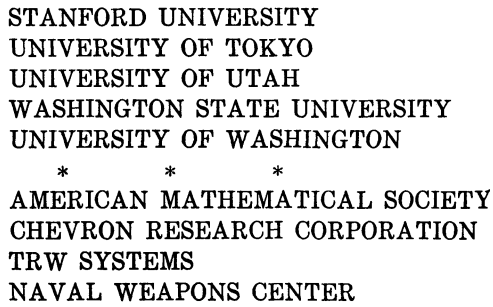

The Supporting Institutions listed above contribute to the cost of publication of this Journal, but they are not owners or publishers and have no responsibility for its content or policies.

Mathematical papers intended for publication in the Pacific Journal of Mathematics should be in typed form or offset-reproduced, double spaced with large margins. Underline Greek letters in red, German in green, and script in blue. The first paragraph or two must be capable of being used separately as a synopsis of the entire paper. It should not contain references to the bibliography. Manuscripts, in duplicate if possible, may be sent to any one of the four editors. Please classify according to the scheme of Math. Rev. 36, 1539-1546. All other communications to the editors should be addressed to the managing editor, Richard Arens, University of California, Los Angeles, California, 90024.

50 reprints are provided free for each article; additional copies may be obtained at cost in multiples of 50 .

The Pacific Journal of Mathematics is published monthly. Effective with Volume 16 the price per volume (3 numbers) is $\$ 8.00$; single issues, $\$ 3.00$. Special price for current issues to individual faculty members of supporting institutions and to individual members of the American Mathematical Society: $\$ 4.00$ per volume; single issues $\$ 1.50$. Back numbers are available.

Subscriptions, orders for back numbers, and changes of address should be sent to Pacific Journal of Mathematics, 103 Highland Boulevard, Berkeley, California, 94708.

PUBLISHED BY PACIFIC JOURNAL OF MATHEMATICS, A NON-PROFIT CORPORATION

Printed at Kokusai Bunken Insatsusha (International Academic Printing Co., Ltd.), 7-17, Fujimi 2-chome, Chiyoda-ku, Tokyo, Japan. 


\section{Pacific Journal of Mathematics}

\section{Vol. 31, No. $2 \quad$ December, 1969}

Efraim Pacillas Armendariz, Quasi-injective modules and stable torsion

classes..........................................

J. Adrian (John) Bondy, On Ulam's conjecture for separable graphs...

Vasily Cateforis and Francis Louis Sandomierski, On commutative rings over which the singular submodule is a direct summand for every module .....

Rafael Van Severen Chacon, Approximation of transformations with continuous

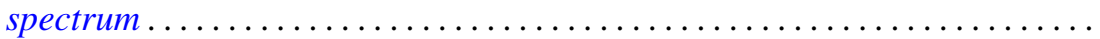

Raymond Frank Dickman and Alan Zame, Functionally compact spaces ...... 303

Ronald George Douglas and Walter Rudin, Approximation by inner

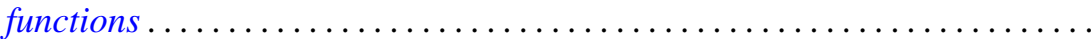

John Walter Duke, A note on the similarity of matrix and its conjugate

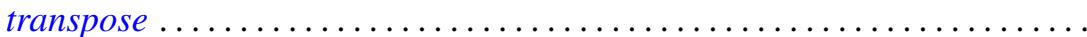

Micheal Neal Dyer and Allan John Sieradski, Coverings of mapping

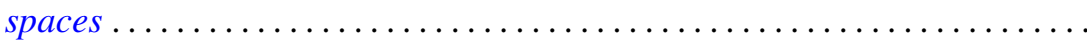

Donald Campbell Dykes, Weakly hypercentral subgroups of finite groups .....

Nancy Dykes, Mappings and realcompact spaces.....................

Edmund H. Feller and Richard Laham Gantos, Completely injective

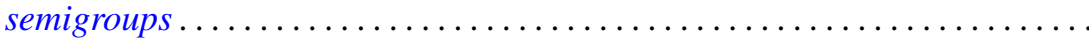

Irving Leonard Glicksberg, Semi-square-summable Fourier-Stieltjes

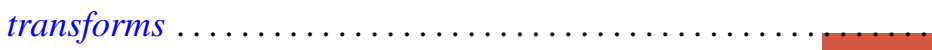

Samuel Irving Goldberg and Kentaro Yano, Integrability of almost cosymplectic structures...

Seymour Haber and Charles Freeman Osgood, On the sum $\sum\langle n \alpha\rangle^{-t}$ and numerical integration ..........................

Sav Roman Harasymiv, Dilations of rapidly decreasing functions ....

William Leonard Harkness and R. Shantaram, Convergence of a sequence of

transformations of distribution functions

Herbert Frederick Kreimer, Jr., A note on the outer Galois theory of rings ...

James Donald Kuelbs, Abstract Wiener spaces and applications to analysis. .

Roland Edwin Larson, Minimal $T_{0}$-spaces and minimal $T_{D}$-spaces...

A. Meir and Ambikeshwar Sharma, On Ilyeff's conjecture .

Isaac Namioka and Robert Ralph Phelps, Tensor products of compact convex sets....

James L. Rovnyak, On the theory of unbounded Toeplitz operators ....

Benjamin L. Schwartz, Infinite self-interchange graphs.......

George Szeto, On the Brauer splitting theorem...

Takayuki Tamura, Semigroups satisfying identity $x y=f(x$,

Kenneth Tolo, Factorizable semigroups .................. 